\title{
Table of statutes
}

Abortion Act 1967 ... 3.16, 11.11, 13.1, 13.3, $13.5,13.12$

s $1(1)(\mathrm{d})$

s $1(3)$

$13.3,13.5$

s 4 ....

$13.3,13.15$

s $5(1)$

13.12

Access to Health Records Act $1990 \quad$...... 4.23

Access to Justice Act 1999 ...................8.2

Administration of Justice Act 1982,

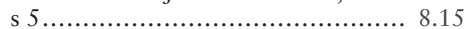

Adoption Act 1976 .......................... 12.2

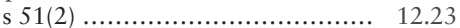

Adoption Act $2002 \quad$...................... 12.28

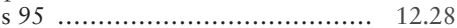

Adoption and Children Act $2002 \ldots \ldots \ldots \ldots \ldots \ldots . . \ldots 12.10,12.29,12.31$ s 95

Adult Support and Protection

(Scotland) Act 2007 ................2.8, 4.8 s $5(3)$ ... 4.8

Anatomy Act 1832

Anatomy Act 1984

Care Act 2014 $17.2,18.4,18.6$ 1.6, 2.4, 2.8, 4.9, 7.22,

$8.12,9.9,15.5$

s 42 $2.8,4.8$

s $73(2)$

2.4

s $81 \ldots \ldots \ldots \ldots \ldots \ldots \ldots \ldots \ldots \ldots \ldots \ldots \ldots \ldots . .6,7.22,8.12,9.9$

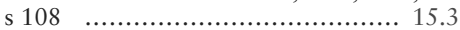

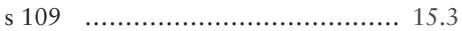

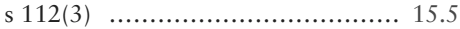

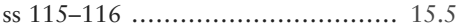

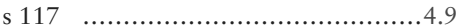

Care Standards Act $2000 \ldots \ldots \ldots \ldots \ldots \ldots .6 .12$

Care Standards Act 2014 ................. 6.22

Children Act 1989 ........ 11.4, 12.10, 14.6, $14.8,14.14$

s 4 . ....

$12.10,14.14$

s 8

$12.12,14.14$

s $31(2)$

14.6

s 100

14.8

Children Act 2004,s $11 \quad$.....................4.1

Children and Families Act 2014 ........ 14.14

Children and Young Persons Act 1933 ... 14.6 s $1(1)$
Compensation Act 2006 $7.6,9.9,10.1$

s 1

.... 7.6

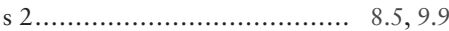

s $3 \ldots \ldots \ldots \ldots \ldots \ldots \ldots \ldots \ldots \ldots \ldots \ldots . .16,10.1,10.10$

Congenital Disabilities(Civil Liability)

Act 1976 ..... 11.7, 11.11, 11.13, 11.14,

$11.16,11.21,12.24$

s $1 \mathrm{~A}$

11.13

s $1(2)$

11.21

s $1(2)(a)$

11.8

s 1 (2)(b)

$11.8,11.11$

s $1(4)$

11.15

s $1(5)$

11.9

s $1(7)$

11.16

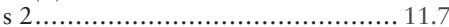

Consumer Protection Act 1987 ... 9.14, 10.1, 10.3, 10.5, 10.15, 11.2,

$11.8,15.15,17.18$

ss $1-2$

$10.5,11.20$

s 3 ..... 11.8

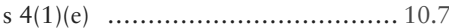

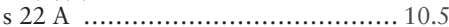

Part I ........................................ 10.1

Consumer Rights Act 2015 ........... 8.7, 10.3

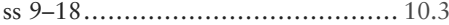

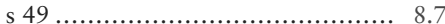

Corneal Tissue Act 1986 ........... 17.2, 17.7

Coroners and Justice Act

$2009 \ldots \ldots \ldots .11 .18,16.3,18.5,19.1,19.5$

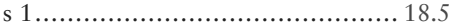

................................... ss $2-4$

18.5

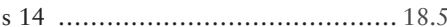

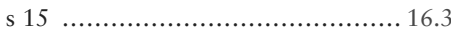

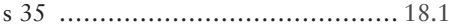

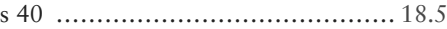

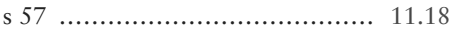

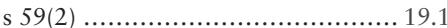

Sch 8 .................................. 18.1

Sch 10 ................................. 18.5

Corporate Manslaughter and Corporate Homicide Act 2007 .......................... 1.17

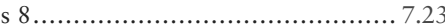

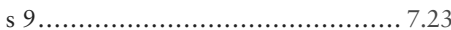




\section{Table of statues}

Counter-Terrorism and Security Act 20154.11 s 26 ........................................ 4.11

Courts and Legal Services Act $1990 \quad \ldots \ldots .8 .3$

Criminal Justice Act 2003 ................. 7.22

Criminal Justice and Courts Act

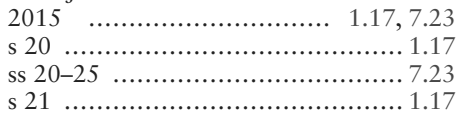

Criminal Law Act 1967

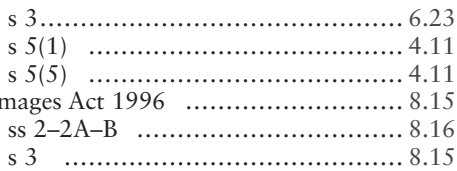

Data Protection Act 1998 ... 4.2, 4.17, 8.12,

$15.17,15.18$

s 7 . 4.23

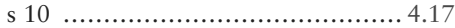

s 13 ..................................... 4.17

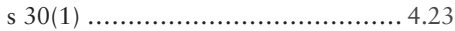

s $51(7) \ldots \ldots \ldots \ldots \ldots \ldots \ldots \ldots \ldots \ldots \ldots \ldots \ldots \ldots \ldots \ldots \ldots \ldots \ldots . . .17$

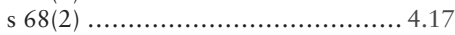

s 69 ...................................... 4.17

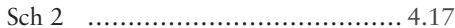

Enduring Powers of Attorney Act 1985 ... 6.15

Enterprise Regulatory Reform Act 2013.

Family Law Act 1996

Family Law Reform Act 1969. 15.10 $14.16,14.21$

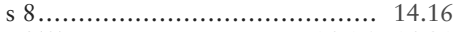
s $8(3)$ $14.16,14.21$

Fatal Accidents Act 1976 8.15

Fatal accidents and Sudden Deaths Inquiry(Scotland) Act 1976 ............ 18.1

Freedom of Information Act 2000 ...... 15.17

Gender Recognition Act 2004........... 17.20

Health Act 1999 s $60 \mathrm{~A}$ 1.14

Health Act 2006 s 60

Health Act 2009 1.18 s 1 ..................................... 2.16

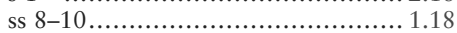

Health and Social Care Act 2001, s 60

Health and Social Care Act 2008 ... 1.8, 6.21 s 145 Part 3

Health and Social Care Act $2012 \ldots \ldots \ldots \ldots \ldots . \ldots 1.10,1.18,9.1,15.18$

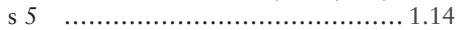

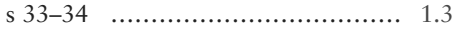

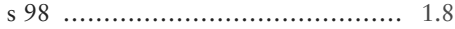

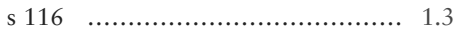

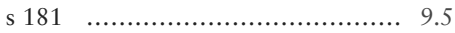

s 182 .................................. 9.5

s 222 $1.2,1.15$

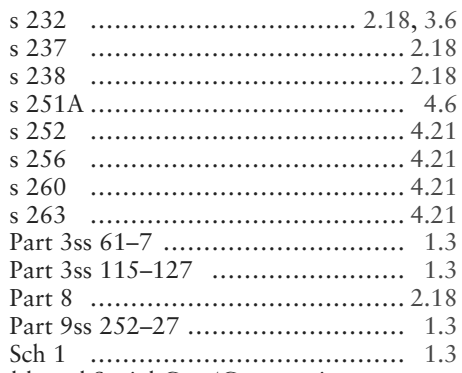

Health and Social Care(Community

Health and Standards) Act 2003 ...... 1.18

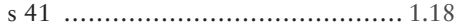

Health and Social Care(Safety and

Quality) Act 2015 ................ 1.1, 4.6

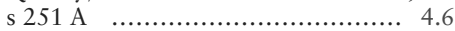

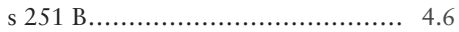

Health Service Commissioners Act

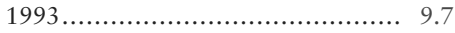

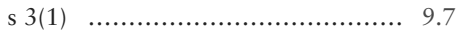

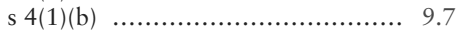

ss $10-12$............................. 9.7

Health Service Commissioners

(Amendment) Act 1996 ............... 9.7

Human Fertilisation and Embryology

Act 1990 ... 1.19, 3.14, 12.1, 12.5, 12.10, $12.11,12.15,12.16,12.21$, 12.27, 13.3, 13.7, 13.12, 13.14, $13.20,18.10$

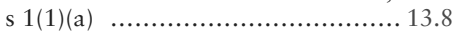

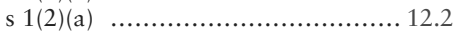

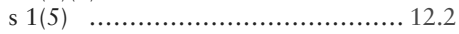

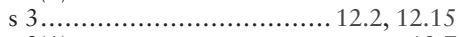

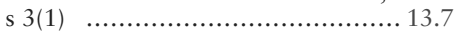

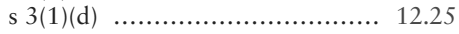

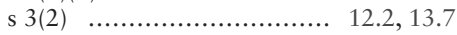

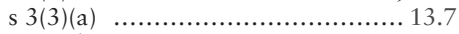

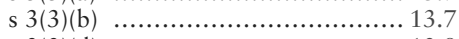

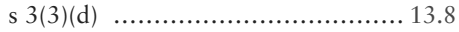

s $3(6) \quad \ldots \ldots \ldots \ldots \ldots \ldots \ldots \ldots \ldots \ldots \ldots \ldots \ldots \ldots \ldots \ldots \ldots \ldots \ldots . .14$

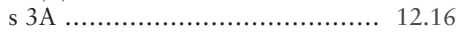

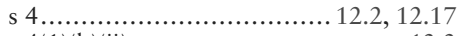

s $4(1)$ (b)(ii) ............................. 12.2

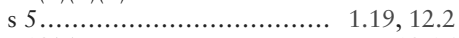

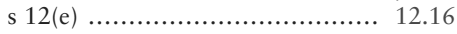

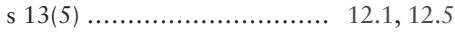

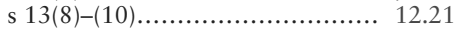

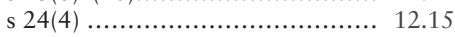

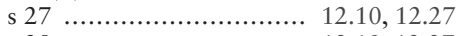

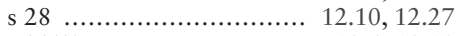

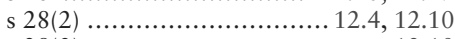

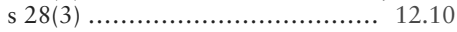

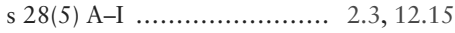

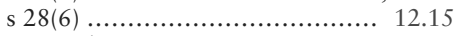

s $28(6)$ (b) .............................. 2.3

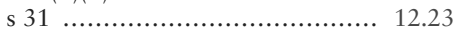

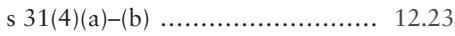




\begin{tabular}{|c|c|}
\hline 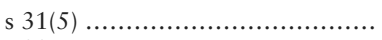 & 12.23 \\
\hline (n) & 12.11 \\
\hline 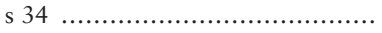 & 12.24 \\
\hline 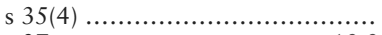 & 12.2 \\
\hline s $37 \ldots \ldots . .$. & 13.1 \\
\hline $37(3) \ldots$ & $\ldots 13$ \\
\hline 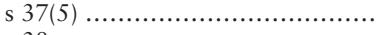 & 13. \\
\hline $38 \ldots \ldots$ & 13. \\
\hline $39-41$ & 12. \\
\hline$\ldots \ldots \ldots \ldots$ & \\
\hline
\end{tabular}

Human Fertilisation and Embryology Act 2008 ..... , $12.2,1211$ $12.12,12.15,12.16,12.19$, $12.21,12.25,12.29,13.1$

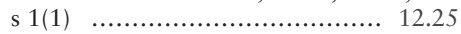

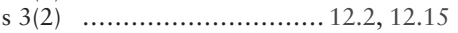

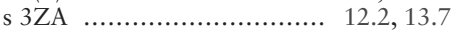

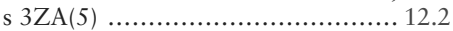

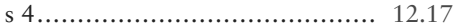

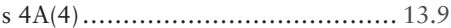

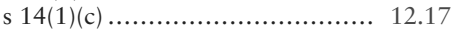

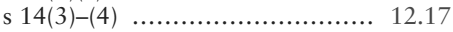

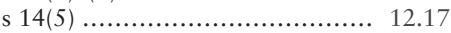

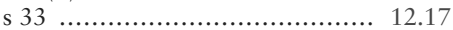

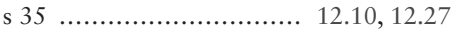

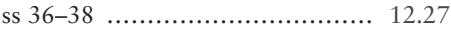

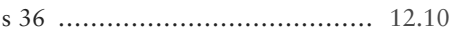

s 36 (d) .................................... 12.27

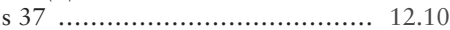

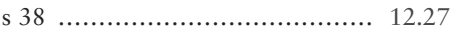

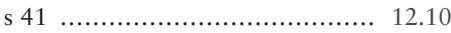

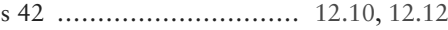

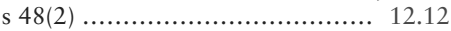

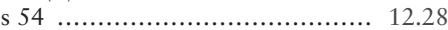

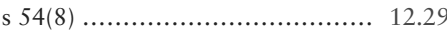

s 59 ................................ 12.29

Sch 2 1ZA ............ 12.19, 12.20, 13.10

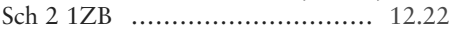

Sch 2 Para $3 A$............................ 13.8

Sch 2 Para 3(4) ......................... 13.7

Sch 3A .................................. 13.8

Sch 3 Para 4A(4) ................... 12.18

Human Fertilisation and Embryology

(Deceased Fathers) Act 2003 ... 2.3, 12.15

Human Reproductive Cloning Act $2001 \ldots \ldots \ldots \ldots . . . .4 .14,12.25,13.8,13.14$

Human Rights Act 1998 ... 2.1, 2.3, 4.2, 6.16, $14.7,19.1$

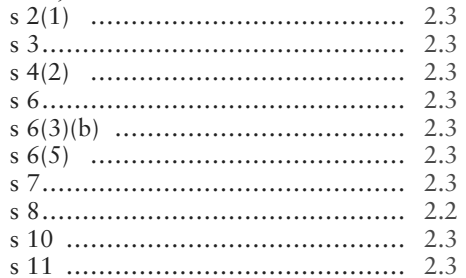

Human Tissue Act 1961 ...... 18.4, 18.6, 18.9

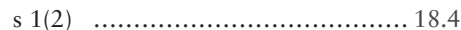

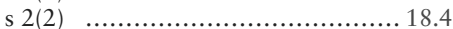

Human Tissue Act 2004 12.16, 12.21, 15.18, 16.4, 17.1, 17.2, 17.9, 17.10, 18.1, 18.4, 18.6,

$18.7,18.8,18.10$

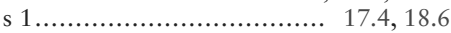

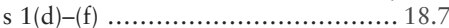

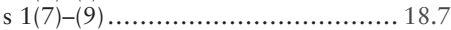

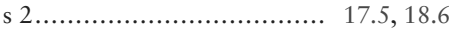

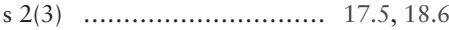

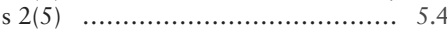

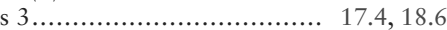

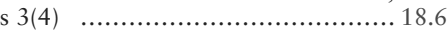

s $3(5) \quad \ldots \ldots \ldots \ldots \ldots \ldots \ldots \ldots \ldots \ldots \ldots \ldots \ldots \ldots \ldots \ldots \ldots . . .4,18.6$

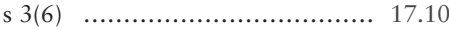

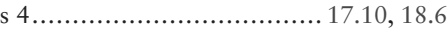

s $5(1) \quad \ldots \ldots \ldots \ldots \ldots \ldots \ldots \ldots \ldots \ldots \ldots \ldots \ldots \ldots .17 .11,18.9$

s $5(2) \quad \ldots \ldots \ldots \ldots \ldots \ldots \ldots \ldots . . . . . . . . . .17 .11,18.9$

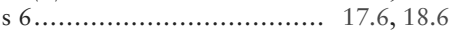

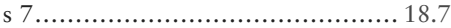

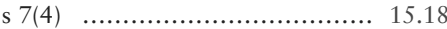

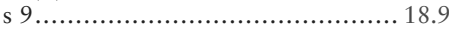

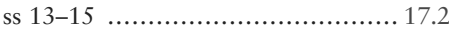

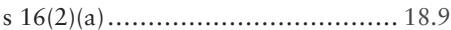

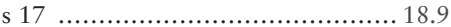

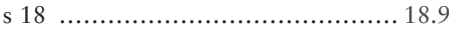

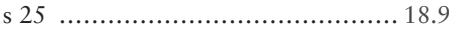

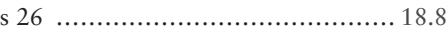

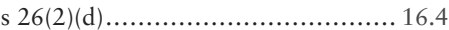

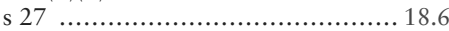

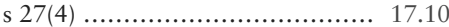

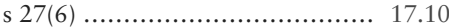

s $27(8) \ldots \ldots \ldots \ldots \ldots \ldots \ldots \ldots \ldots . . .17 .10,18.6$

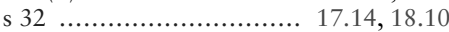

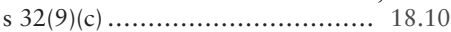

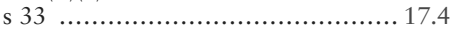

s 43 ...................................... 17.9

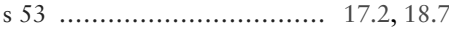

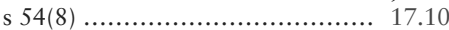

Sch $1 \quad \ldots \ldots \ldots \ldots \ldots \ldots \ldots . . . \ldots \ldots . .2,18.6,18.7$

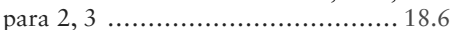

Sch 2 ........................ 17.2, 18.6

Human Tissue Act (Northern Ireland)

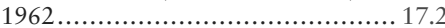

Human Tissue (Scotland) Act $2006 \quad$....... 17.1

Human Transplantation (Wales) Act

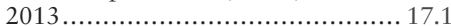

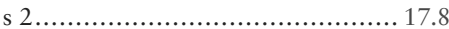

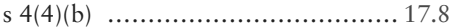

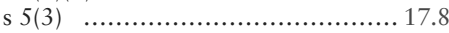

Infanticide Act $1938 \ldots \ldots \ldots \ldots \ldots \ldots \ldots . . . . . . . .11 .18$

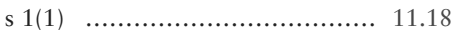

Infant Life(Preservation) Act

$1929 \ldots \ldots \ldots \ldots \ldots \ldots \ldots \ldots . . . . . . . . .13 .12,13.16$

Injury Prevention, Rehabilitation and Compensation Act 2001 ....................... 9.13

s 32 ....................................... 9.13

Inquiries Act $2005 \quad$.......................... 9.2

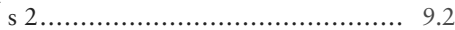




\section{Table of statues}

s 15

Law of Property Act 1925, s 184 16.2

Law Reform(Contributory

Negligence) Act 1945 7.20

Law Reform (Personal Injuries) Act 1948, s $2(4)$ 8.15

Legal Aid, Sentencing and Punishment of Offenders Act 2012 ...... 2.10, 8.2, 8.18, $9.1,10.2$

s 10 .......

s $10(3)$ 8.2

s 21 8.2

Sch 1 Part I ........................... 8.2

Limitation Act 1980

s 11 8.9

s 14 8.9

Local Government and Public Involvement in Health Act 2007

s 221 9.5

Medical Act 1969 .......................... 1.8

Medical Act 1983 .............. 1.8, 6.18, 11.21

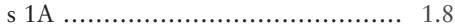

s 5 ....... 1.11

Pt III(ss 19-29) ................... 1.8, 6.18

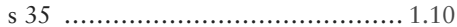

s $35 \mathrm{C}(2) \quad \ldots \ldots \ldots \ldots \ldots \ldots \ldots \ldots \ldots \ldots \ldots \ldots \ldots \ldots \ldots \ldots \ldots . .14$

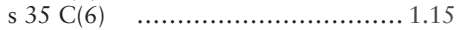

s $35 \mathrm{C}(8) \quad \ldots \ldots \ldots \ldots \ldots \ldots \ldots \ldots \ldots \ldots \ldots \ldots \ldots \ldots \ldots \ldots . .14$

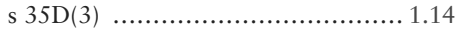

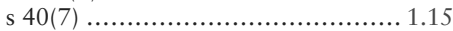

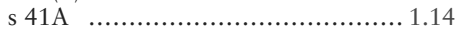

s 49 .................................... 1.8

Medicines Act 1968 ..................... 10.11

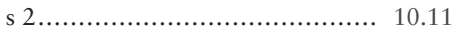

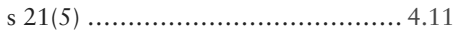

s 28 .................................. 10.11

Mental Capacity Act 2005 ...... 4.4, 4.7, 5.2, $6.1,6.4,6.12,6.23,7.23$, $11.4,11.21,12.15,13.18$ 14.14, 14.21, 15.10, 15.11, $17.9,19.1,19.17,19.18$ $19.19,19.21$

s $1(5)$ 11.4

s $1(6)$ 11.4

s $2 \ldots$

$11.21,14.21$

s $2(1)$ $6.4,11.4$

s $2(3)$ (b) s $2(5)$ $5.2,11.4$

s $3 \ldots \ldots$ $4.4,6.4$ s $3(2)$

s $4 \ldots$ 6.4

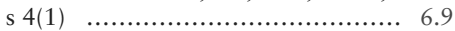
s $4(3)(4)$ 6.9 s $4(5)$ $6.10,19.18$

s $4(6)$ $6.9,11.4$

s $4(6)(a)(b)$ 19.19

s $4(7)$

s $4(10)$

s 5 . $4.7,5.2,6.9,11.4$ $5.2,6.1,6.7,6.8,11.4$ s 6 6.10

s 9 ss $9-14$ $6.8,6.15$

s 10 19.19

s $11(8)(a)$ 19.19

ss $12-14$

$19.19,19.21$

s 15 19.19

s 16 6.16

ss 17 $6.12,6.16$

s 20 6.16 6.16

s 21 6.16 $5.2,6.14$

ss $24-26$ 19.19

s 24(2)

s 25 $2.7,5.3,6.14$

s $25(1)$ $2.7,5.2,6.14$

s $25(3)(4)$ $6.14,19.19$

s $25(5)$

s $25(6)$

s 26 .......

s 26(1) 6.14

s 30 ..... 19.19

s 30(4)

s 30(2)-(4)

s 31

s $31(5)(6)$.

s 32

s $32(8)$

s $32(9)$

s 33 $6.14,19.19$ $2.7,6.14$

s 34 6.14

$6.10,15.11$ 15.11 6.10 6.10

s $42(4)(5)$........

s 44

ss $46-61$

s $64(5)$

$6.10,15.11$ 6.10

$6.10,15.11$

Sch A1 15.12

Mental Health Act 1983 ...... 4.4, 6.18, 6.19, $6.23,7.23,11.21,14.21$

Pt II(ss 2-34) 6.18

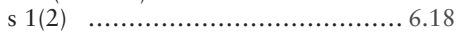

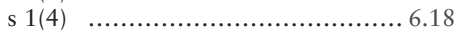

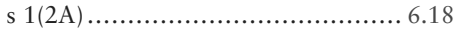

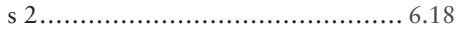

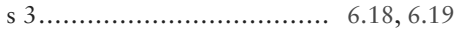

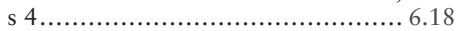

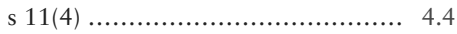

s 13 ................................... 4.4

Pt IV(ss 56-64) ....................... 6.18

s 57 ......................................... 6.19

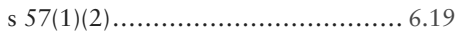

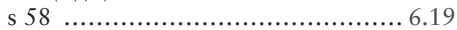

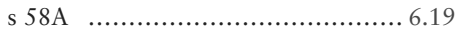

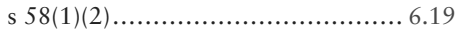

s $63 \ldots \ldots \ldots \ldots \ldots \ldots \ldots \ldots \ldots \ldots . .6 .5,6.18,6.19$

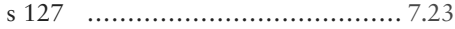

s 145 .................................... 6.18

Mental Health Act 2007 ........ 2.9, 6.18, 14.21

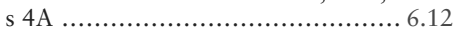

s 4B ......................................... 6.12 
s 50 6.1

Sch A1 6.12

Sch $1 \mathrm{~A}$ 6.12

Misuse of Drugs Act 1971 ............... 4.9

National Assistance Act $1948 \quad$........... 2.4 s $47 \ldots \ldots \ldots \ldots \ldots \ldots \ldots \ldots \ldots \ldots \ldots \ldots \ldots \ldots \ldots \ldots \ldots . .22$

National Assistance(Amendment) 1951... 6.22

National Health Service Act 1977 ......... 18.2

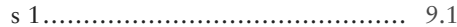

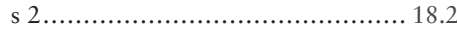

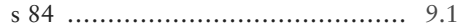

National Health Service Act 2006 . ... 2.17, 2.20, 4.9, 15.8, $15.17,15.18$

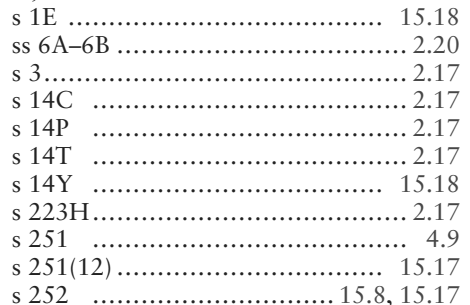

National Health Service Reform and Health Care Professions Act 2002,

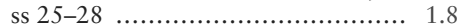
NHS Redress Act $2006 \quad \ldots \ldots . . .9 .1,9.3,9.18$

Nurses, Midwives and Health Visitors Act 1997,s 16 11.19

Offences Against the Person Act $1861 \ldots \ldots \ldots \ldots .6 .22,11.18,13.2,13.20$

s 20 6.22

s 23 11.18 s 58 $13.2,13.20$

Oregon Death with Dignity Act 1994. $19.5,19.12$

Police and Criminal Evidence Act 1984.

ss $8-14$ 4.9

Sch 1 4.9

Protection of Freedoms Act 2012 ..... 15.18 Protection of Life During Pregnancy Act(Ireland) 2013
Public Bodies Act 2011 1.19

Public Health(Control of Diseases)

Act 1984 $4.4,4.9,6.21$

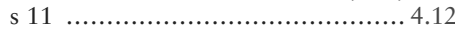

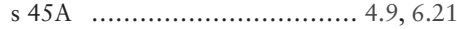

ss $45 \mathrm{~B}-\mathrm{E}$........................... 6.21

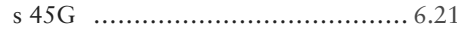

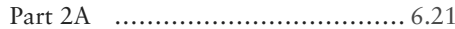

Public Interest Disclosure Act 1998 ..... 1.5

Road Traffic Act 1998 s 172

Sale of Goods Act 1979 .................... 10.8

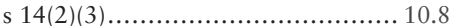

Senior Courts Act 1981 ................... 8.12

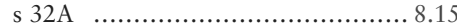

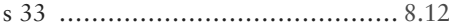

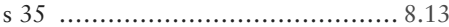

Serious Crime Act 2015 .......... 4.11, 13.4 ss $70-75 \ldots \ldots \ldots \ldots \ldots \ldots \ldots \ldots \ldots \ldots . . .11$

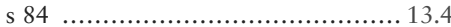

Sexual Offences Act 1956 ............. 14.20

Sexual Offences Act 2003 ...... 14.19, 14.20

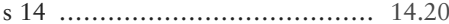

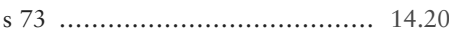

Social Services and Well-being (Wales) Act 2014 .............. 2.8, 4.8

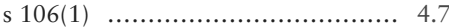

Suicide Act 1961 .......... 19.1, 19.4, 19.22

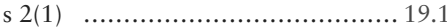

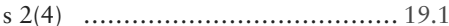

Surrogacy Arrangements Act 1985 12.29

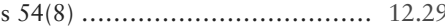

Terrorism Act 2000 s 19 4.11

Tribunals of Inquiry(Evidence)

Act 1921

Unfair Contract Terms Act 1977 s 2

Vaccine Damage Payments Act 1979

Vermont Patient Choice and Control and End of Life Act 2013 19.5

Washington State Death with Dignity Act 2009 
Margaret Brazier and Emma Cave - 9781526153425 Downloaded from manchesterhive.com at $04 / 26 / 2023$ 11:34: 99 AM 